\title{
Shock Wave Therapy Enhances Angiogenesis through VEGFR2 Activation and Recycling
}

\author{
Tien-Hung Huang, ${ }^{1}$ Cheuk-Kwan Sun, ${ }^{7}$ Yi-Ling Chen, ${ }^{1}$ Ching-Jen Wang, ${ }^{3,4}$ Tsung-Cheng Yin, ${ }^{4}$ \\ Mel S Lee, ${ }^{4^{*}}$ and Hon-Kan Yip ${ }^{1,2,3,5,6^{*}}$
}

${ }^{1}$ Division of Cardiology, Department of Internal Medicine, Kaohsiung Chang Gung Memorial Hospital, Kaohsiung, Taiwan; ${ }^{2}$ Institute for Translational Research in Biomedicine, Kaohsiung Chang Gung Memorial Hospital, Kaohsiung, Taiwan; ${ }^{3}$ Center for Shockwave Medicine and Tissue Engineering, Kaohsiung Chang Gung Memorial Hospital, Kaohsiung, Taiwan; ${ }^{4}$ Department of Orthopedic Surgery, Kaohsiung Chang Gung Memorial Hospital and Chang Gung University College of Medicine, Kaohsiung, Taiwan; ${ }^{5}$ Department of Medical Research, China Medical University Hospital, China Medical University, Taichung, Taiwan; ${ }^{6}$ Department of Nursing, Asia University, Taichung, Taiwan; and ${ }^{7}$ Department of Emergency Medicine, E-Da Hospital, I-Shou University School of Medicine for International Student, Kaohsiung, Taiwan

\begin{abstract}
Although low-energy shock wave (SW) is adopted to treat ischemic diseases because of its pro-angiogenic properties, the underlying mechanism remains unclear. This study is aimed at testing whether SW-induced angiogenesis may be through endothelial vascular endothelial growth factor receptor 2 (VEGFR2) signaling and trafficking. Phosphorylation of VEGFR2Akt-eNOS axis and production of nitric oxide (NO) were determined in human umbilical vein endothelial cells (HUVECs) treated with SW. Carotid artery in ob/ob mice was treated with SW before evaluation with sprouting assay. Critical limb ischemia was induced in ob/ob mice to evaluate blood flow recovery post-SW treatment. Tube formation and migration assays were also performed with/without SW treatment in the presence/absence of SU5416 (VEGFR2 kinase inhibitor) and siRNA-driven silencing of VEGFR2. Chloroquine was used for disrupting endosome, and Rab 1 la controlling slow endocytic recycling was silenced with siRNA in vitro. Following SW treatment, augmented ligand-independent phosphorylation in VEGFR2-Akt-eNOS axis and endogenous NO production, increased cellular migration and tube formation and elevated sprouting of carotid artery and blood flow in ischemic limb in ob/ob mice were noted. Moreover, SU5416 and VEGFR2 silencing both inhibited SW-induced angiogenesis. SW-induced angiogenesis, accompanied by increased VEGFR2 protein expression without transcriptional change, was suppressed by chloroquine and Rab 1 la silencing. We concluded that SW enhanced angiogenesis via ligand-independent activation of VEGFR2 and further prolonged angiogenesis through endosome-to-plasma membrane recycling in endothelial cells. Online address: http://www.molmed.org
\end{abstract} doi: $10.2119 / \mathrm{molmed} .2016 .00108$

\section{INTRODUCTION}

Shock wave (SW) therapy is a noninvasive mechanical treatment strategy that has been applied clinically for decades. While high-energy SW is widely used to splinter stones over the urinary tract, low-energy SW is commonly adopted for the treatment of musculoskeletal disorders (1-4). Recently, low-energy SW has been shown to be a promising therapeutic option in the treatment of erectile dysfunction $(5,6)$. A number of studies have attempted to extend SW application to other diseases
*MSL and H-KY contributed equally to this paper.
Address correspondence to Hon-Kan Yip, Division of Cardiology, Department of Inter-
nal Medicine, Kaohsiung Chang Gung, Memorial Hospital, 123, Dapi Road, Niaosung
Dist., Kaohsiung City, 83301, Taiwan. Phone: + 886-7-7317123; Fax: + 886-7-7322- 402;
E-mail: han.gung@msa.hinet.net.
Submitted April 22, 2016; Accepted for publication November 11, 2016;
Published Online (www.molmed.org) December 6, 2016.

*MSL and H-KY contributed equally to this paper.
Address correspondence to Hon-Kan Yip, Division of Cardiology, Department of Inter-
nal Medicine, Kaohsiung Chang Gung, Memorial Hospital, 123, Dapi Road, Niaosung
Dist., Kaohsiung City, 83301, Taiwan. Phone: + 886-7-7317123; Fax: + 886-7-7322-402;
E-mail: han.gung@msa.hinet.net.
Submitted April 22, 2016; Accepted for publication November 11, 2016;
Published Online (www.molmed.org) December 6, 2016.

*MSL and H-KY contributed equally to this paper.
Address correspondence to Hon-Kan Yip, Division of Cardiology, Department of Inter-
nal Medicine, Kaohsiung Chang Gung, Memorial Hospital, 123, Dapi Road, Niaosung
Dist., Kaohsiung City, 83301, Taiwan. Phone: + 886-7-7317123; Fax: + 886-7-7322- 402;
E-mail: han.gung@msa.hinet.net.
Submitted April 22, 2016; Accepted for publication November 11, 2016;
Published Online (www.molmed.org) December 6, 2016.

*MSL and H-KY contributed equally to this paper.
Address correspondence to Hon-Kan Yip, Division of Cardiology, Department of Inter-
nal Medicine, Kaohsiung Chang Gung, Memorial Hospital, 123, Dapi Road, Niaosung
Dist., Kaohsiung City, 83301, Taiwan. Phone: + 886-7-7317123; Fax: + 886-7-7322- 402;
E-mail: han.gung@msa.hinet.net.
Submitted April 22, 2016; Accepted for publication November 11, 2016;
Published Online (www.molmed.org) December 6, 2016.

*MSL and H-KY contributed equally to this paper.
Address correspondence to Hon-Kan Yip, Division of Cardiology, Department of Inter-
nal Medicine, Kaohsiung Chang Gung, Memorial Hospital, 123, Dapi Road, Niaosung
Dist., Kaohsiung City, 83301, Taiwan. Phone: + 886-7-7317123; Fax: + 886-7-7322- 402;
E-mail: han.gung@msa.hinet.net.
Submitted April 22, 2016; Accepted for publication November 11, 2016;
Published Online (www.molmed.org) December 6, 2016.

*MSL and H-KY contributed equally to this paper.
Address correspondence to Hon-Kan Yip, Division of Cardiology, Department of Inter-
nal Medicine, Kaohsiung Chang Gung, Memorial Hospital, 123, Dapi Road, Niaosung
Dist., Kaohsiung City, 83301, Taiwan. Phone: + 886-7-7317123; Fax: + 886-7-7322- 402;
E-mail: han.gung@msa.hinet.net.
Submitted April 22, 2016; Accepted for publication November 11, 2016;
Published Online (www.molmed.org) December 6, 2016.

*MSL and H-KY contributed equally to this paper.
Address correspondence to Hon-Kan Yip, Division of Cardiology, Department of Inter-
nal Medicine, Kaohsiung Chang Gung, Memorial Hospital, 123, Dapi Road, Niaosung
Dist., Kaohsiung City, 83301, Taiwan. Phone: + 886-7-7317123; Fax: + 886-7-7322- 402;
E-mail: han.gung@msa.hinet.net.
Submitted April 22, 2016; Accepted for publication November 11, 2016;
Published Online (www.molmed.org) December 6, 2016.

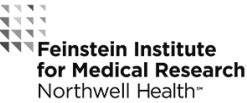

Feinstein Institute
for Medical Research Northwell Health or injuries using different animal models, including burn wounds, myocardial infarction and critical limb ischemia (7-11). Despite clear demonstration of SW-induced angiogenesis in those studies, the exact mechanisms remain poorly understood.

In the processes of stimulation generated by blood flow-induced shear stress, vascular endothelial growth factor receptor 2 (VEGFR2) acts as a mechanotransducer to transmit activated signal through ligand-independent phosphorylation, thereby leading to vasodilation via endothelial nitric oxide synthase (eNOS) activation (12) and plays a predominate role in VEGFR2-induced angiogenesis (13). Similar to laminar shear stress in endothelial cells, SW has been reported to lead to 
mechanosensory complex formation and Akt/eNOS phosphorylation (14). On the other hand, VEGFR2 undergoes constitutive endosome-to-plasma membrane recycling in endothelial cells (15). VEGFR2 recycling accompanies dephosphorylation of VEGFR2 through endosomal vesicles (16). Thus, balance of VEGFR2 recycling is important for signal in and out. Receptor traffic also has been reported to be affected by external environmental factors including infection, aging and degenerative diseases (17) as well as stimulation by fluid shear stress in renal proximal tubular cells (18). However, whether SWinduced angiogenesis is associated with VEGFR2 recycling is still unknown.

The present study, therefore, tested the hypothesis that low-energy SW treatment may trigger the VEGFR2-Akt-eNOS axis and further prolong angiogenesis through endocytic VEGFR2 recycling in endothelial cells.

\section{MATERIALS AND METHODS}

\section{Ethics}

All animal experimental procedures were approved by the Institute of Animal Care and Use Committee at Kaohsiung Chang Gung Memorial Hospital (Affidavit of Approval of Animal Use Protocol No. 2014032701 and No. 2015061503) and performed in accordance with the National Research Council of the National Acadamies Guide for the Care and Use of Laboratory Animals (Eighth Edition, 2011).

\section{Shock Wave Treatment}

Focused SW with adjustable levels between 0.10 to $0.15 \mathrm{~mJ} / \mathrm{mm}^{2}$ was produced from HMT Evotron Shock Wave Therapy Device (HMT High Medical Technologies). Before SW treatment, HUVECs were pre-washed once by PBS and filled with PBS in a culture dish without any bubble. Probe of the SW-producing device was applied vertically on the top of the culture dish with ultrasound gel (Figure 1A). Culture dishes were purchased from TPP (surface area size $60.1 \mathrm{~cm}^{2}$ ).

\section{Cell Culture}

HUVEC cells were purchased from Bioresource Collection and Research Center (BCRC). Culture medium (endothelial cell medium, ECM) was purchased from SciencCell. HUVEC cells between passage 4 to 6 were used in this study and were grown at $37^{\circ} \mathrm{C}$ under $5 \%$ $\mathrm{CO}_{2}$ in $0.1 \%$ gelatin pre-coated culture dish.

\section{Transfection of siRNAs and Treatment with SU5416, Cycloheximide and Chloroquine}

Oligonucleotides of siRNAs, SU5416 (S8442), and chloroquine (C6628) were all purchased from Sigma-Aldrich. Three siRNA sequences to human VEGFR2 were SASI_Hs01_00073462 + SASI_Hs01_00073462_AS (No.1),SASI_ Hs01_00073461 + SASI_Hs01_00073461_ AS (No.2) and SASI_Hs01_00073463 + SASI_Hs01_00073463_AS (No.3). Three siRNA sequences to the human Rab11a were SASI_Hs01_00126208 + SASI_Hs01_00126208_AS (No.1),SASI_ Hs01_00126207 + SASI_Hs01_00126207_ AS (No.2) and SASI_Hs01_00126206 +SASI_Hs01_00126206_AS (No.3). Transfection of siRNAs (50 nmol/L) into HUVECs was accomplished with TransIT-X2 dynamic delivery system (Mirus) in complete medium for $3 \mathrm{~d}$, according to the manufacturer's recommendation. Addition of SU5416 was performed prior to tube formation assay. Cycloheximide (CHX, $10 \mu \mathrm{g} / \mathrm{mL})$ and chloroquine (CHQ, $5 \mu \mathrm{mol} / \mathrm{L}$ and $20 \mu \mathrm{mol} / \mathrm{L}$ ) were added into the medium $2 \mathrm{~h}$ post-SW treatment. For another $26 \mathrm{~h}$ incubation, treated HUVECs were subjected to tube formation assay.

\section{Nitric Oxide Detection}

Intracellular nitric oxide (NO) production was determined using specific cell permeable fluorescent probe 4-amino-5-methylamino-2', '7'-difluorofluorescein diacetate (DAF-FM DA; Molecular Probes). After SW treatment, $5 \mu \mathrm{mol} / \mathrm{L}$ of DAF-FM diacetate was added into HUVECs in serum- and growth factor-free M199 basal medium, defined as basal medium (BM), and incubated at $37^{\circ} \mathrm{C}$ for $40 \mathrm{~min}$. Green fluorescent derivate was converted in the presence of $\mathrm{NO}$ and was counterstained with Hoechst 33342. The fluorescence signals were analyzed using fluorescence microscopy (Olympus Bx51).

\section{PKH26 and PKH67 Staining}

PKH26 (MINI26) and PKH67 (MINI67) were all purchased from Sigma-Aldrich. For general cell membrane labeling, control and SW-treated HUVECs were stained with PKH26 (red fluorescence dye) and PKH67 (green fluorescence dye), respectively. Cells were labeled according to the manufacturer's protocol. After membrane labeling, both cells were mixed together with equal numbers, and were subjected to tube formation assay. The fluorescence signals were captured using fluorescence microscopy (Olympus IX51).

\section{Cell Proliferation Assay}

After SW treatment, HUVECs were plated onto a 96-well plate. Rate of cell proliferation in 4 consecutive days was determined by incubation with $0.5 \mathrm{mg}$ / $\mathrm{mL}$ MTT (AMRESCO) for $4 \mathrm{~h}$ and the precipitated formazan was dissolved in DMSO. The supernatant was assessed at a wavelength of $540 \mathrm{~nm}$.

\section{Wound-Healing Migration Assay and Transwell Migration Assay}

For the wound-healing migration assay, HUVECs were plated onto culture insert (iBidi $\mathrm{GmbH}$ ). After full confluence of culture cells, the insert was removed and replaced by serum- and growth factor-free M199 basal medium (BM) for migration assay. After $16 \mathrm{~h}$ incubation, images of cell migration were retrieved using an inverted phase contrast microscope (Olympus IX51), and were quantitated by WimScratch (Wimasis Image Analysis). For the transwell migration assay, HUVECs were plated on 6-well transwells with $8-\mu \mathrm{m}$ pore-size insert (Falcon) precoated with $0.1 \%$ gelatin. $3 \times 10^{5} \mathrm{SW}$ treated HUVECs or control HUVECs were seeded on insert, $3 \times 10^{5}$ normal 
A
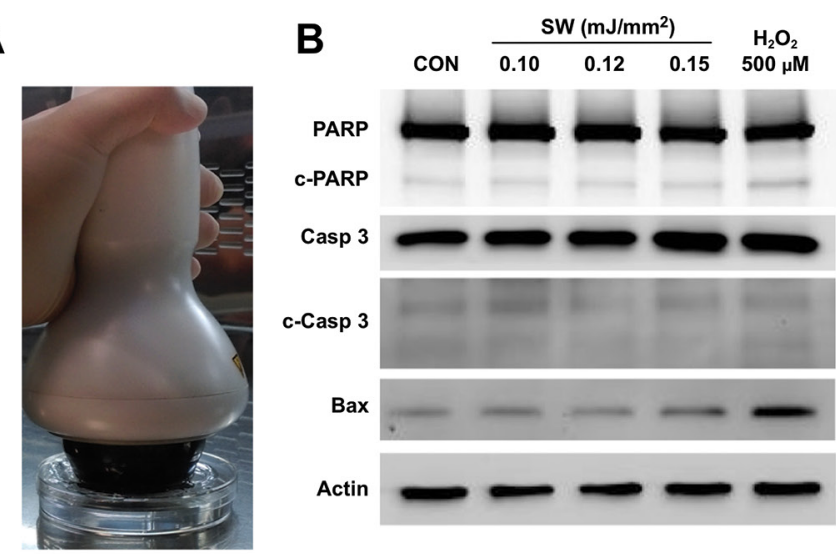

C

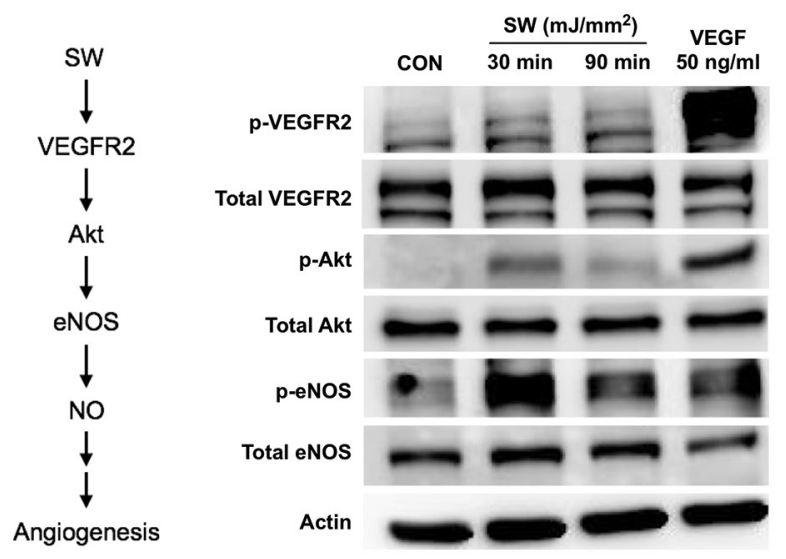

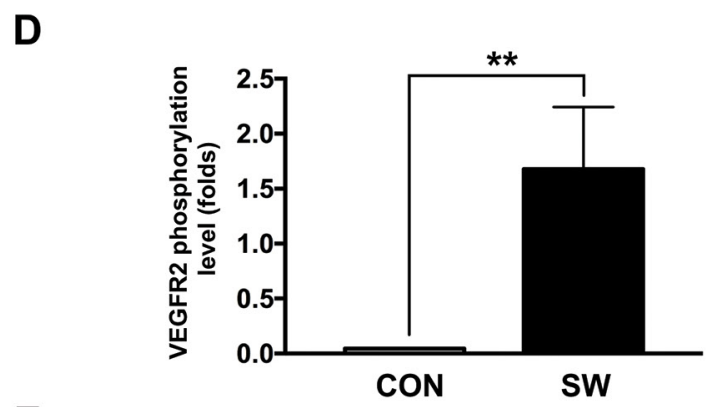

E
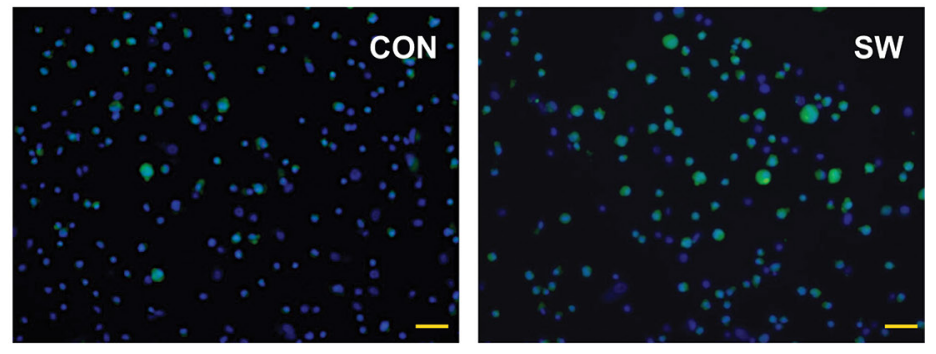

$\mathbf{F}$

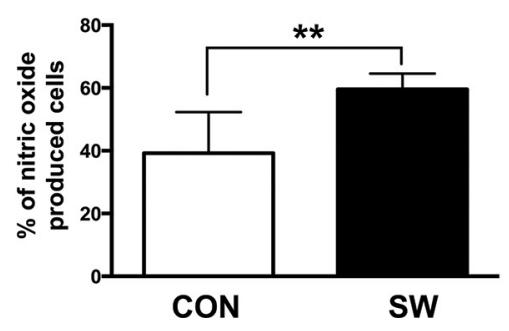

Figure 1. Activation of VEGFR2-Akt-eNOS signaling pathway by shock wave treatment in HUVECs. (A) Demonstration of shock wave delivery to culture dish. (B) Expressions of cellular apoptosis-related proteins in HUVECs $28 \mathrm{~h}$ post-SW treatment assessed by Western blot, including cleavage PARP (C-PARP), cleavage caspase 3 (c-Casp 3) and Bax. Treatment of $\mathrm{H}_{2} \mathrm{O}_{2}(500 \mu \mathrm{mol} / \mathrm{L}$ ) used as positive control. (C) Illustration showed that SW-induced angiogenesis may be achieved through VEGFR2-Akt-eNOS signaling pathway. Phosphorylation of VEGFR2, Akt and eNOS in HUVECs at 30 min and 90 min post-SW treatment compared with those in the control (CON) without SW treatment and with vascular endothelial growth factor A (VEGFA) treatment ( $50 \mathrm{ng} / \mathrm{mL}$ ) in serum- and growth factor-free medium being used as positive control. (D) Quantification of VEGFR2 phosphorylation in HUVECs without (that is, CON) or with SW treatment ( $\mathrm{C}=4$ in each group). (E) Representative fluorescent images of DAF-FM diacetate-treated HUVECs post-nitric oxide activation without (CON) or with SW treatment. Comparison of the percentage of fluorescence-positive cells between the two groups ( $n=7$ in each group). ( $F$ ) Measurement of carotid artery contraction without (CON) and with SW treatment. Left pane: Potassium chloride (KCl)-induced vessel contraction. Right panel: Phenylephrine (PE)-induced vessel contraction. Data shown as means \pm S.D. ${ }^{* *} P<0.005$ and ${ }^{*} P<0.05$ determined by Student $t$ test.

HUVECs were seeded on the bottom of the culture dish, and were cultured with M199 medium ( $1 \%$ FBS) at $37^{\circ} \mathrm{C}$ with $5 \%$ $\mathrm{CO}_{2}$. After $16 \mathrm{~h}$, nonmigrated cells were removed from the upside of insert, and migrated cells in the underside were fixed by methanol and further counted by the hematoxylin staining.

\section{Sprouting Assay}

Adult male leptin-deficient mice (ob/ob), a well-established model of type 2 diabetes mellitus, were purchased from National Laboratory Animal Center and divided randomly into two groups (control group and SW group). The SW group received SW at an energy of 0.12 $\mathrm{mJ} / \mathrm{mm}^{2}, 200$ impulses at a frequency of $2 \mathrm{~Hz}$ on both sides of the neck and were allowed to recover for $2 \mathrm{~d}$ post treatment. For ex vivo angiogenesis assay, SWtreated carotid arteries were separated and cut into small pieces of approximately $1 \mathrm{~mm} \times 1 \mathrm{~mm}$, and further rinsed by PBS. Carotid arteries were carefully placed on Matrigel-coated $(10 \mu \mathrm{L}) \mu$-slide (iBidi $\mathrm{GmbH}$ ) and further covered with $20 \mu$ l Matrigel, followed by incubation for $30 \mathrm{~min}$ at $37^{\circ} \mathrm{C}$. After gelation, $30 \mu \mathrm{L}$ of complete medium was added for another 5-d incubation. The medium was replaced every $2 \mathrm{~d}$. Seven days post-SW treatment, images of sprouting were retrieved using an inverted phase contrast microscope (Olympus IX51). Both sprout area and mean of sprout front 
distance were quantitated by WimSprout (Wimasis Image Analysis).

\section{Mouse Critical Limb Ischemia Model and Measurement of Blood Flow with Laser Doppler Flowmetry}

Adult male ob/ob mice were randomly divided into two groups (control group and SW group) and anesthetized preoperatively with $3 \%$ isoflurane prior to CLI induction ( $\mathrm{n}=4$ for each group). Under sterile conditions, the left femoral artery, small arterioles and circumferential femoral artery were exposed and ligated over their proximal and distal portions before removal. Shock wave at an energy of $0.12 \mathrm{~mJ} / \mathrm{mm}^{2}, 200$ impulses with frequency of $2 \mathrm{~Hz}$. was administered at $\mathrm{d} 1$ and 7 after induction of critical limb ischemia (CLI). Fourteen days post-CLI, the mice were placed in a supine position and the blood flow detected in both inguinal areas by a laser Doppler flowmetry (moorLDLS, Moor Co.). The ratio of flow in left leg (ischemic) to that of right leg (normal) was calculated.

\section{RNA Extraction and Real-Time RT-qPCR}

Total RNA was extracted using Trizol reagent (Invitrogen), following the protocol of the manufacturer. Reverse transcription was performed by Transcriptor First Strand cDNA Synthesis Kit (Roche). Expression of VEGFR2 was determined by using Quantifast SYBR Green PCR kit (Qiagen) with the forward primer of 5'-GTGACCAACATGGAGTCGTG -3' and the reverse primer of 5'-TGCTTCACAGAAGACCATGC -3', and normalized by GAPDH expression with the forward primer of 5'-GACAGTCAGCCGCATCTTCTT- $3^{\prime}$ and the reverse primer of 5'-CCAATACGACCAAATCCGTTGAC-3'. Triplicate assays were performed for each sample on Step OnePlus machine (ABI).

\section{Western Blot}

Protein was extracted from cultured cells by using cell scraper and RIPA buffer with protease and phosphatase inhibitor. Approximate amounts of protein were subjected to SDS-PAGE and transferred to membranes. The membranes were incubated with the primary antibody, including actin (1:1000, MAB1501, Millipore), VEGFR2, phospho-VEGFR2 (Tyr 1175) (1:500, 3770, Cell Signaling), Akt (1:1000, 9272, Cell Signaling), phospho-Akt (Ser 473) (1:1000, 9271, Cell Signaling), eNOS (1:1000, 9572, Cell Signaling) and phospho-eNOS (1:500, 9571, Cell Signaling). Horseradish peroxidase (HPR)-conjugated secondary antibody was used for binding the primary antibody. Immunoreactive bands were visualized by enhanced chemiluminescence (ECL, Amersham Biosciences) and protein bands were quantified using ImageJ software.

\section{Tube Formation Assay}

HUVEC cells were transfected transiently with scramble siRNA or VEGFR2 siRNA or Rab11a. Three days posttransfection, transfected HUVEC cells were seeded on Matrigel-coated $\mu$-slide (iBidi $\mathrm{GmbH}$ ) with serum- and growth factor-free M199 basal medium (BM) for tube formation assay. Treatment of SU5416 (S8442, Sigma-Aldrich), or chloroquine (CHQ) (C6628, Sigma-Aldrich) was performed prior to tube formation assay. After $6 \mathrm{~h}$ incubation, images of network generated from tube formation were retrieved using an inverted phase-contrast microscope (Olympus IX51), and were quantitated by WimTube (Wimasis image analysis). Parameters, including total tube lengths, branch points and loop numbers, were quantitated and transformed into percentage relative to control.

\section{Collection of Conditioned Medium and Enzyme-Linked Immunosorbent Assay (ELISA) for VEGFA}

Conditioned medium was collected from HUVECs with or without SW treatment after tube formation assay (50 $\mu \mathrm{L}$ /each well in $\mu$-slide) and further centrifuged at $290 \mathrm{~g}$ for $5 \mathrm{~min}$ to eliminate unnecessary cells. Conditioned medium was applied in two assays.
One was a tube formation assay for the determination of conditioned medium-induced angiogenesis in HUVECs, while the other was quantification of secreted VEGFA by ELISA (eBioscience, BMS277) in conditioned medium according to the manufacturer's recommendation.

\section{Statistical Analysis}

GraphPad Prism software (ver. 6) (GraphPad Software) was used for statistical analyses and data plotting. Results shown are mean with SD. Significance of difference among different groups was analyzed by unpaired Student $t$ test and post-test for a linear trend. A probability value $<0.05$ was considered statistically significant.

\section{RESULTS}

\section{Low-Energy Shock Wave Treatment Displayed Harmless Effects on HUVECs}

To determine whether HUVECs displayed cellular apoptosis in response to SW treatment, we applied three different low-energy levels $(0.10,0.12$ and $0.15 \mathrm{~mJ} / \mathrm{mm}^{2}$ ) to determine the apoptotic and DNA-damaged protein expression by Western blot post-SW treatment (Figure $1 \mathrm{~B}$ ). $\mathrm{H}_{2} \mathrm{O}_{2}$-treated HUVECs were used as positive control to verify the protein expression of cellular apoptosis and DNA damage. All SW-treated HUVECs maintained low expression of cleavage fragment of poly ADP-ribose polymerase (PARP) and cleavage fragment of caspase 3 as compared to control. However, increased Bax protein expression was observed at the level of $0.15 \mathrm{~mJ} / \mathrm{mm}^{2}$. Optimal conditions for SW treatment were found to be 0.12 $\mathrm{mJ} / \mathrm{mm}^{2}, 200$ impulses at a frequency of $2 \mathrm{~Hz}$. Therefore, we selected the level of $0.12 \mathrm{~mJ} / \mathrm{mm}^{2}$ for the following experiments.

\section{Shock Wave Activated Ligand- Independent VEGFR2-Akt-eNOS Signaling Pathway}

To determine whether our shock wave (SW) condition $\left(0.12 \mathrm{~mJ} / \mathrm{mm}^{2}\right.$, 
200 pulses, $2 \mathrm{~Hz}$ ) is able to stimulate the VEGFR2-Akt-eNOS axis, we examined the phosphorylation status of VEGFR2, Akt and eNOS in HUVECs by Western blots (Figure 1C). Addition of recombinant VEGF protein was used as positive control. In serum- and growth factor-free M199 basal medium (BM), SW evoked strong VEGFR2, Akt and eNOS phosphorylation at $30 \mathrm{~min}$ and further reduced at $90 \mathrm{~min}$ as compared with the phosphorylation status in the control. To examine the production of NO, DAF-FM diacetate was used as an indicator to assess nitric oxide (NO) production post-SW treatment (Figure 1D). The number of nitric oxide-converted fluorescent cells in the SW group was significantly higher than that in the control group $(P=0.0025)$ (Figure 1E). These results indicated that ligand-independent activation of VEGFR2-Akt-eNOS axis, which plays a predominant role in angiogenesis, was revealed in HUVECs post SW treatment.

\section{SW-Induced Angiogenesis Revealed a Dose-Dependent Increase}

A few tube structures were founded in serum- and growth factor-free M199 basal medium (BM) as compared with that of complete medium. To evaluate whether SW conducted angiogenesis with a dose-dependent increase, different conditions (below $0.12 \mathrm{~mJ} / \mathrm{mm}^{2}$, 200 impulses with frequency of $2 \mathrm{~Hz}$ ) in BM for SW delivery were applied in HUVECs and further examined by tube formation assay (Figure 2A-B). By comparing control to SW-treated HUVECs with increasing energy and pulses, there were significant progressive increases in angiogenesis, quantitated by the parameters of tube length $(P$ for trend $=0.0092)$, branch point $(P$ for trend $=$ 0.0189 ) and loop number $(P$ for trend $=0.0287$ ) (Figure 2C). Specifically, SWtreated HUVCEs with $0.12 \mathrm{~mJ} / \mathrm{mm}^{2}$ (200 impulses) displayed a significantly higher capacity of angiogenesis than that in the control. This condition, therefore, was used in the following experiments. To determine whether SW affects the capacity of tube formation, SW-treated HUVECs pre-labeled with PKH26 (red fluorescence) were mixed with an equal number of normal HUVECs pre-treated with PKH67 (green fluorescence) to monitor for network formation (Figure 2D). The results showed co-constructure from normal and SW-treated HUVECs. Therefore, we demonstrated that SW-treated HUVECs expressed normal tube formation function compatible with that of neighboring endothelial cells in BM.

\section{Characteristic Difference between Normal and SW-Treated HUVECs}

To examine whether the property of endothelial cells was altered by SW treatment, we performed wound healing, transwell migration and cellular proliferation assays with control and SW-treated HUVECs. In both wound healing and transwell migration assay,
A

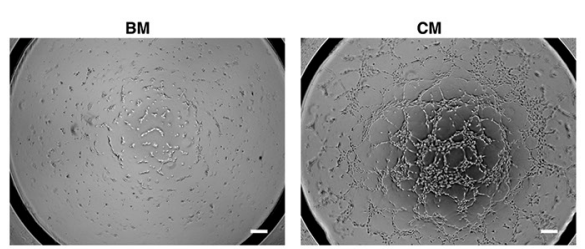

B
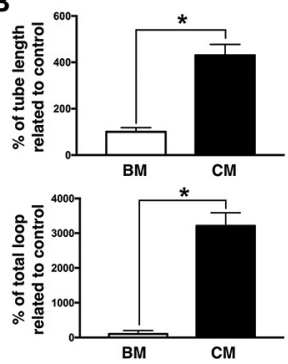

C
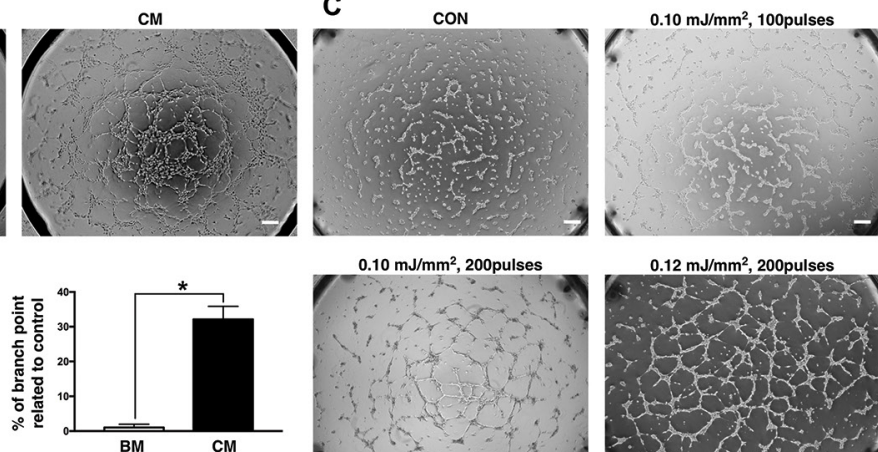

$0.10 \mathrm{~mJ} / \mathrm{mm}^{2}, 200$ pulses

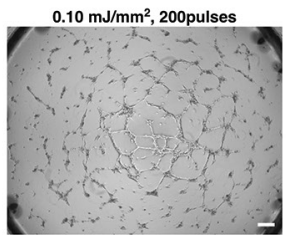

D

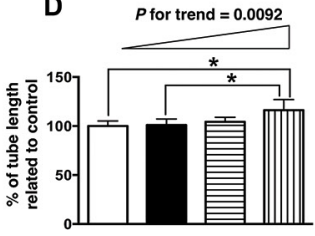

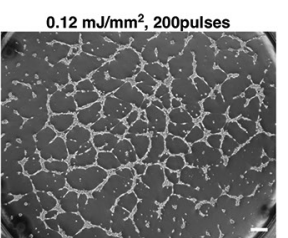

$P$ for trend $=0.0189$

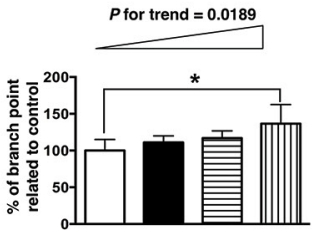

E
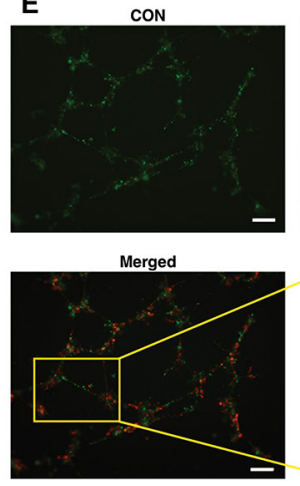

$P$ for trend $=0.0287$

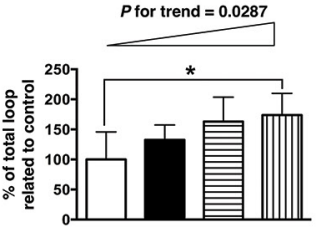

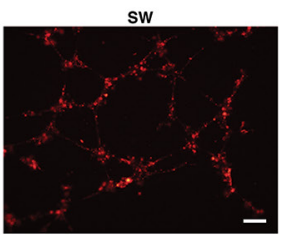

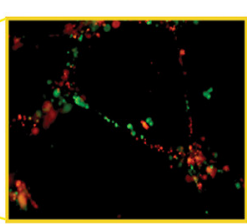

믈 coN

- $0.10 \mathrm{~mJ} / \mathrm{mm}^{2}, 100$ impulses 曰 $0.10 \mathrm{~mJ} / \mathrm{mm}^{2}, 200$ impulses $0.12 \mathrm{~mJ} / \mathrm{mm}^{2}, 200$ impulses

Figure 2. Shock wave (SW)-induced angiogenesis in vitro. (A) Representative images of tube formation assay with basal medium (BM, defined as serum- and growth factors-free) and complete medium (CM). (B) Parameters of tube formation assay, including tube length, branch point and the number of loops ( $n=5$ in each group). (C) Representative images of tube formation assay in control (CON) group and a dose-dependent increase of SW treatment in HUVECs (scale bar, $200 \mu \mathrm{m}$ ). (D) Parameters of tube formation assay, including tube length, branch point and the number of loop ( $n=5$ in each group). $P$ for trend in those parameters showing a dose-dependent increased order. (E) Control (CON) and SW-treated HUVECs labeled with PKH67 (green) and PKH26 (red), respectively. Representative images of fluorescence signals (scale bar, $100 \mu \mathrm{m}$ ). Merged image with magnification indicated by yellow line showing amalgamation in tube formation assay. Data shown as mean \pm S.D. ${ }^{*} P<0.05$ determined by Student $t$ test. 
A
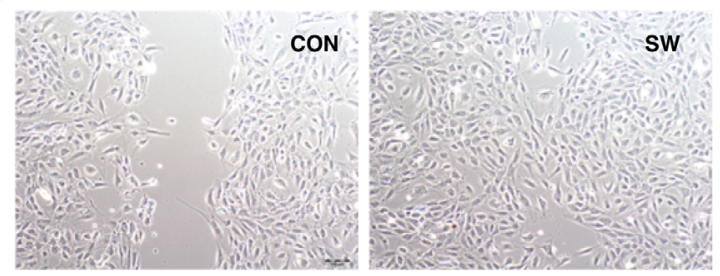

B
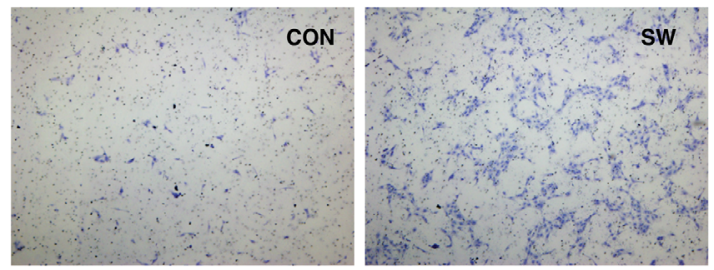

C

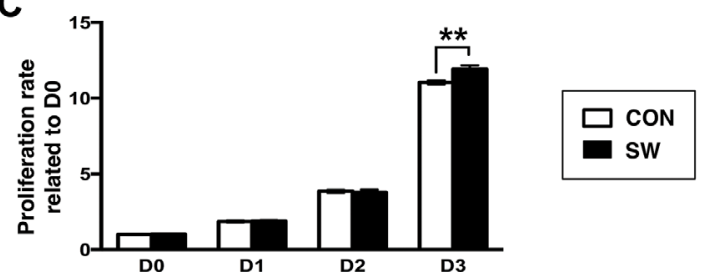

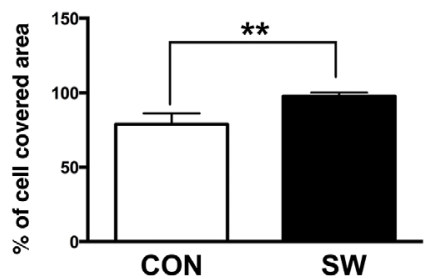

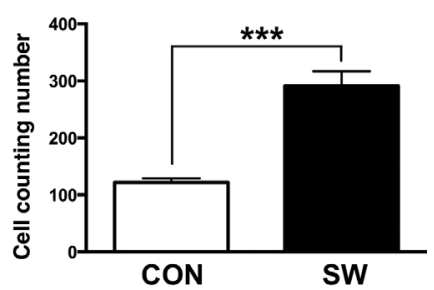

Figure 3. Enhancement of cellular migration and proliferation by shock wave (SW) treatment. (A) Representative images of wound healing assay without (CON) and with SW treatment. Computation of cell-covered area by WimScratch image analysis for cell motility determination ( $n=4$ in each group). (B) Representative images of transwell migration assay without (CON) and with SW treatment. Counting of hematoxylin-stained migrated cells by ImageJ for the cell motility determination ( $n=5$ in each group). (C) For cellular proliferation determination, HUVECs collected from d 0 to d 3 post-SW treatment subjected to MTT assay ( $n=4$ in each group/per d). Data shown as means \pm S.D. ${ }^{*} P<0.05,{ }^{* *} P<0.005,{ }^{* * *} P<0.0005$ by Student $t$ test.

SW-treated HUVECs showed significantly higher migration than control $(P=0.0031$ and 0.0002) (Figure 3A-B). In cellular proliferation assay, there were no significance differences during the first $2 \mathrm{~d}$, but a higher proliferation rate was displayed in SW-treated HUVECs at d $3(P=0.0007)$ (Figure 3C).

\section{Angiogenesis Enhanced by SW Ex Vivo and In Vivo}

To examine the capacity of angiogenesis ex vivo and in vivo, we performed carotid artery sprouting assay and Matrigel plug assay to quantitate the difference in angiogenesis with and without SW treatment. In carotid sprouting assay, mice with SW treatment exhibited significantly higher sprout areas and sprout distances than those in the control
$(P=0.0191, P=0.0077)$ (Figure 4A-B). In the critical limb ischemia model (Figure 4C-D), ob/ob mice with SW treatment $(n=4)$ also showed higher recovered blood flow than those in the control $(\mathrm{n}=4)(P=0.0384)$.

\section{SW-Induced Angiogenesis Mediated by VEGFR2}

To clarify the mechanism of SW-induced angiogenesis, we compared the effects of control-conditioned medium (CM) to those of SW-treated CM in tube formation assay, but there was no significant difference in angiogenesis (Figure 5A-B). Furthermore, quantification of vascular endothelial growth factor A (VEGFA) under both conditions also displayed no significant difference (Figure 5C). The results, therefore, suggested that SW-induced angiogenesis was not caused by secreted factors including VEGFA. VEGFR2 has been known to be a critical signaling receptor involved in angiogenesis. To clarify the role of VEGFR2 in SW-induced angiogenesis, VEGFR2-inhibitor and VEGFR2specific siRNAs were applied in HUVECs. Treatment of SU5416 (a selective inhibitor of tyrosine kinase activity of VEGFR2) significantly inhibited SW-induced angiogenesis (Figure 6B-C) $(n=7$, all parameters showed $P<0.0005)$. Furthermore, knockdown of VEGFR2 protein expression was conducted by three different siRNAs, No.1 and No.2 were used for VEGFR2 knockdown (Figure 6D). Inhibition of SW-induced angiogenesis was also demonstrated in HUVECs transfected with the two siRNAs (Figure 6E-F) $(\mathrm{n}=6$, all parameters showed $P<0.05)$. Therefore, we assumed that VEGFR2 protein expression plays a critical role in $\mathrm{SW}$-induced angiogenesis.

\section{Recycling of Endocytic VEGFR2 Increased post-SW Treatment}

Interestingly, we found that VEGFR2 protein expression showed a significant elevation $28 \mathrm{~h}$ post-SW treatment (Figure 7A) (n= 4, $P=0.0032)$ without $V E G F R 2$ mRNA increase (Figure 7B). We wonder whether an increase of VEGFR2 protein expression was determined by endosome-to-plasma membrane recycling. To address the role of SW in endosome recycling specifically, a mediator of recycling endosome, Rab11a, which regulated late endocytic recycling (19), was silenced by specific siRNAs. Knockdown of Rab11a protein expression was conducted by three different siRNAs (No.1, No.2, and No.3) and the latter two siRNAs were used for Rab11a inhibition (Figure 7C). Angiogenesis was found to be inhibited in HUVECs transfected with those two siRNAs (Figure 7D-E).

To locate the elevated VEGFR2 expression in HUVECs, cell lysates were fractionized into three different compartments (membrane, cytosol and nucleus proteins). Although VEGFR2 protein expression 
A

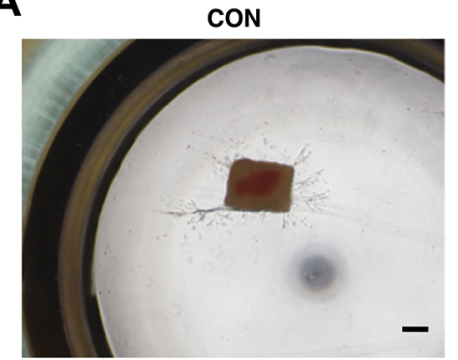

CON (after processing)

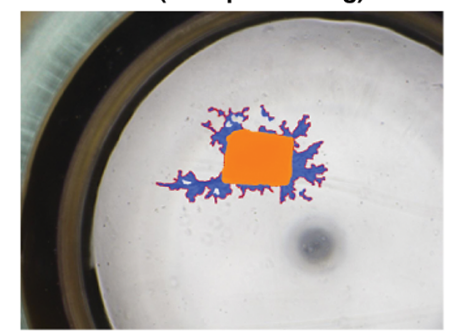

B

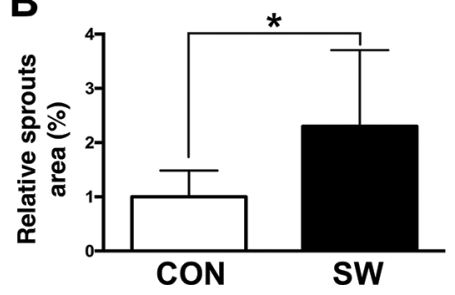

sw

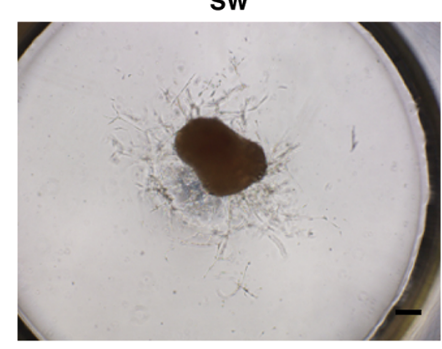

SW (after processing)
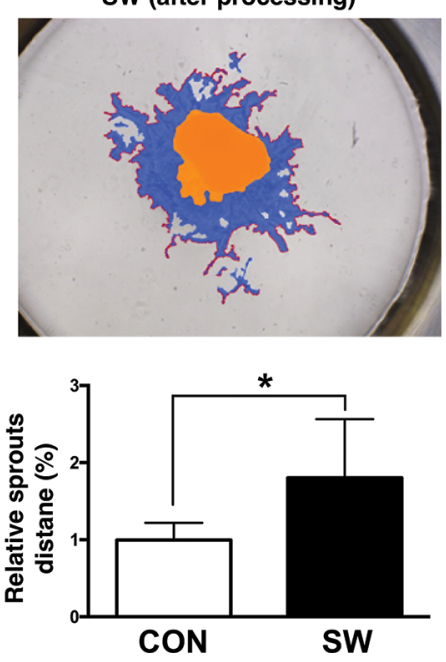

C
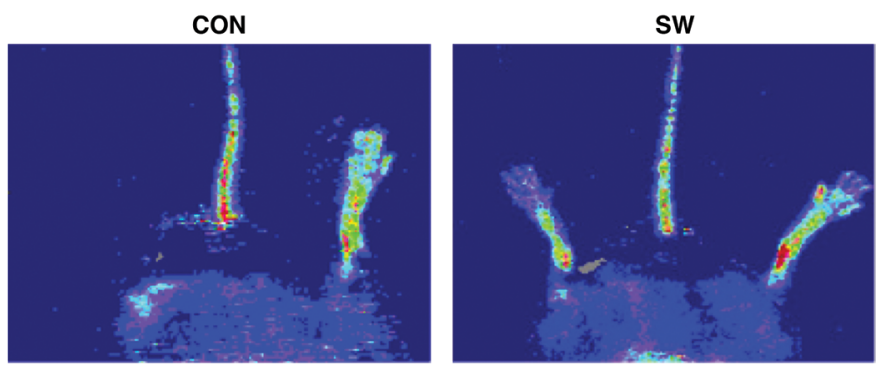

D

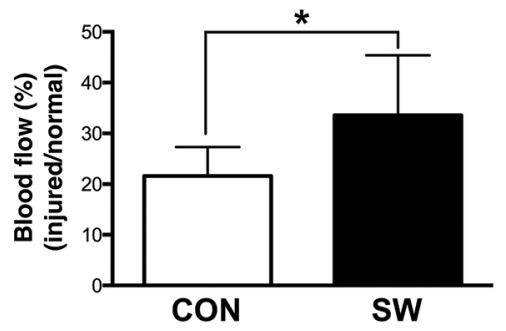

Figure 4. Shock wave (SW)-induced angiogenesis ex vivo and in vivo. (A) Representative sprouting morphology in ob/ob mice carotid artery with $(n=8)$ and without $(n=9)$ SW treatment (scale bar, $200 \mu \mathrm{m})$. Upper panel: Original images; Lower panel: Computer-processed images by WimSprout image analysis for sprouting determination. (B) Parameters of sprouting assay, including sprout area and sprout distance. (C) Representative images of laser Doppler flowmetry in ob/ob mice without (CON) and with SW treatment $14 \mathrm{~d}$ post-critical limb ischemia (CLI). (D) Measurement of blood flow post-CLI without (CON, $n=9)$ and with $(n=7)$ SW treatment by laser Doppler flowmetry. Data presented as mean \pm S.D. ${ }^{*} P<0.05$ determined by Student $t$ test.

did not change after the first $4 \mathrm{~h}$ of SW administration in the membrane protein compartment, it was increased $28 \mathrm{~h}$ post-SW treatment (Figure 8A), implying that SW-induced VEGFR2 recycling may sustain more than $1 \mathrm{~d}$.

To examine whether endosome abnormalities affect SW-induced angiogenesis, HUVECs were treated with cycloheximide (CHX) and chloroquine (CHQ) post-SW for tube formation assay. Cycloheximide (CHX) was applied for inhibition of protein synthesis. On the other hand, chloroquine (CHQ) has been reported to induce an abnormal endocytic pathway including blocking of endosome acidification as well as swelling of the endosome $(20,21)$. Thus, VEGFR2 protein expression in cytosol compartment was increased dramatically post-CHQ treatment, possibly as a result of endosome disruption (Figure 8B). Furthermore, $\mathrm{SW}$-induced angiogenesis as reflected in tube formation was found to be inhibited by CHQ treatment (Figure 8C-D). By contrast, treatments with $\mathrm{CHX}$ alone and $\mathrm{CHX}+\mathrm{CHQ}$ did not disrupt tube formation in the condition of complete medium (Figure 8E-F). Taken together, the results indicated that part of SW-induced angiogenesis may be caused by VEGFR2 endosome recycling.

\section{DISCUSSION}

The major findings of this study are that SW not only enhanced angiogenesis but also prolonged its duration through VEGFR2 activation following recycling in endothelial cells. We successfully established an optimal SW setting for the induction of angiogenesis in vitro, ex vivo and in vivo. The prolonged SW-induced angiogenesis was found to be attributable to an increased number of VEGFR2 from endosome-plasma-membrane recycling regardless of protein synthesis. Importantly, this was an advantageous method to characterize an endocytic VEGFR2 recycling pathway in endothelial cells in response to SW treatment.

Diabetic patients exhibit impaired angiogenesis through inhibition of nitric oxide (NO) synthesis and display damaged vessel wall structure via formation of advanced glycation end products (AGEs) (22). Diabetic foot, which is one of the common vascular complications of diabetes, 
A

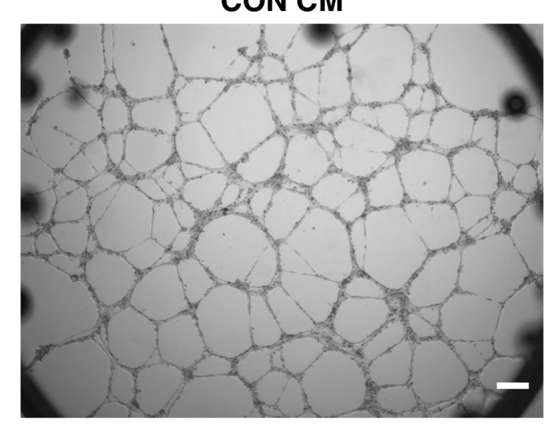

B
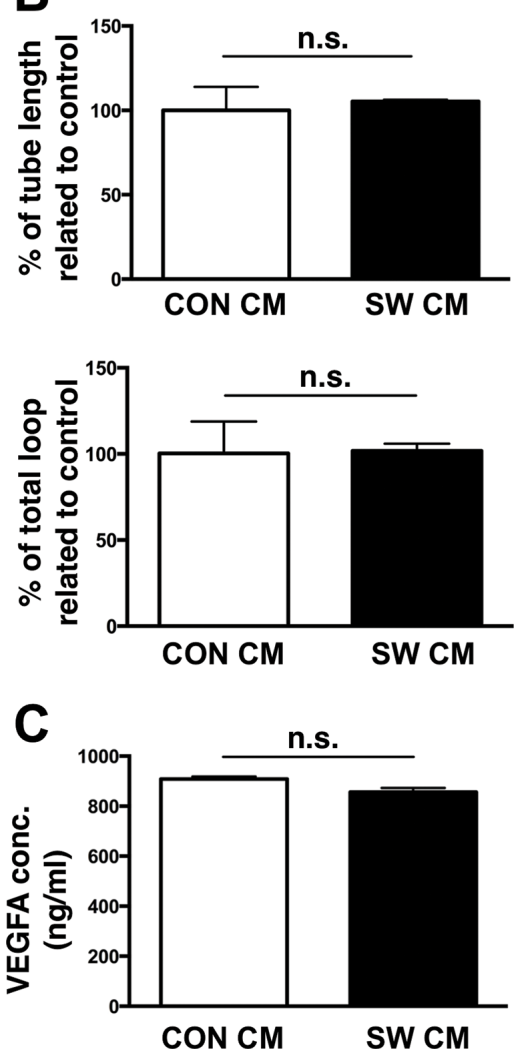

Figure 5. No vascular endothelial growth factor-A (VEGFA) increase post-shock wave (SW) administration. (A) Conditioned medium from control (CON CM) and SW-treated HUVECS (SW CM) applied to normal HUVECs for observation of tube formation ( $n=3$ in each group). (B) Parameters of tube formation assay, including tube length, branch point and loop number. (C) VEGFA in the conditioned medium from control and SW-treated HUVECs quantitated by ELISA ( $n=4$ in each group). Data shown as mean \pm S.D. No significance (n.s.) determined by Student $t$ test.

has been known to accompany severe pathological impairments in peripheral arteries and neurons (23). Recently, SW therapy has been applied in patients with diabetic foot ulcer for improving the rate of wound healing $(24,25)$. In this study, we also found that SW treatment in ob/ob
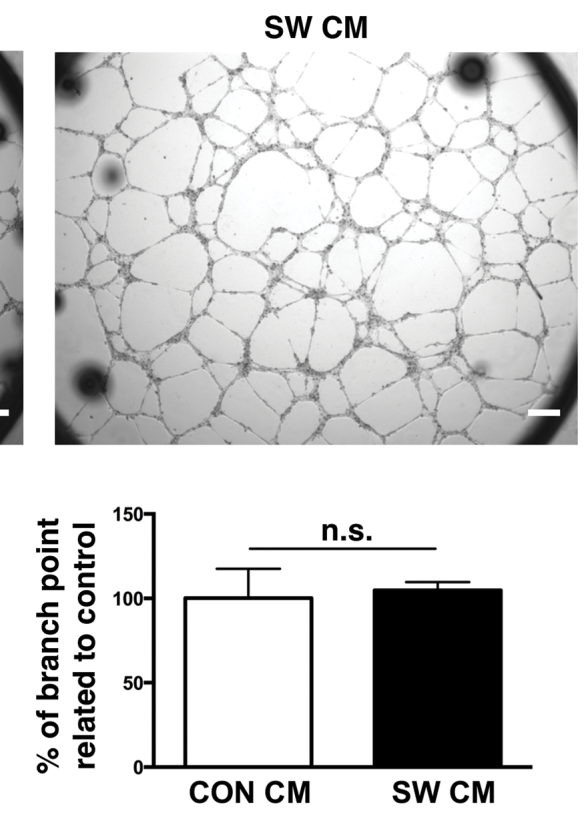

. 
A
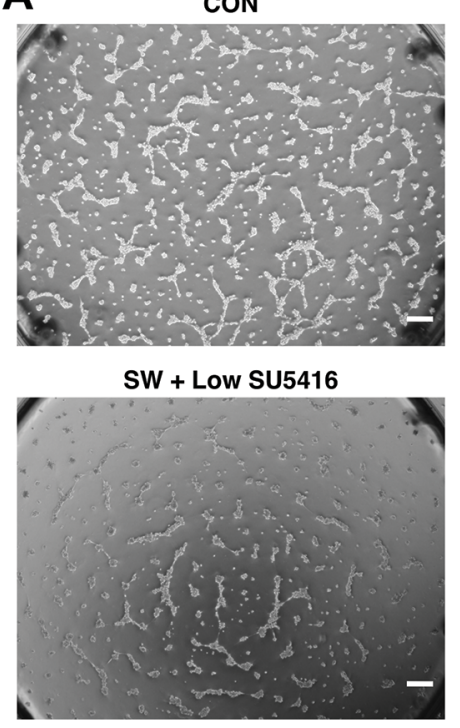

B
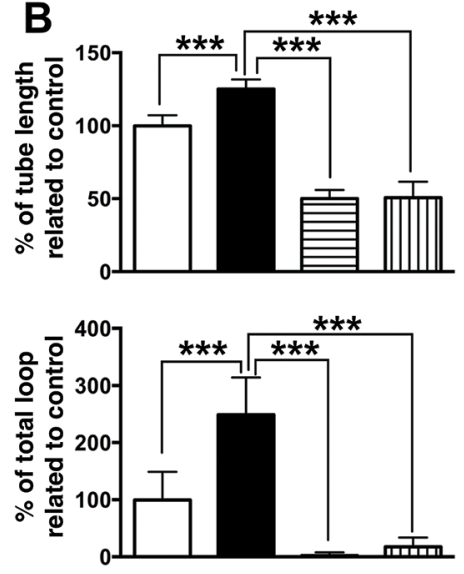

C

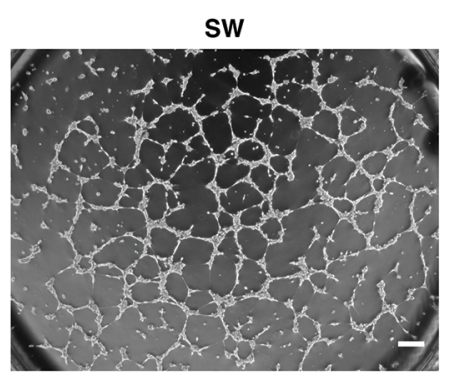

SW + High SU5416
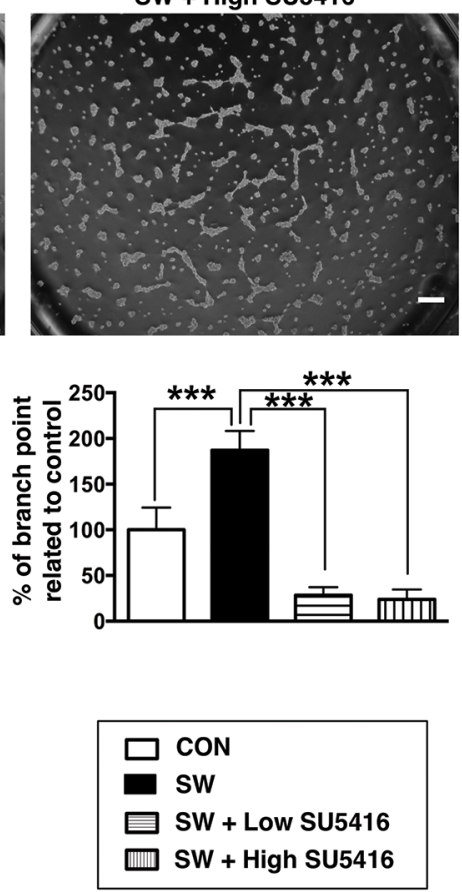

D

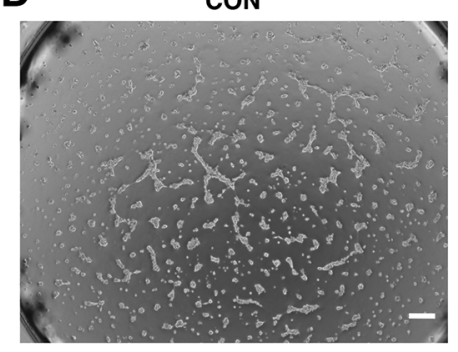

SW + No.1 siRNA

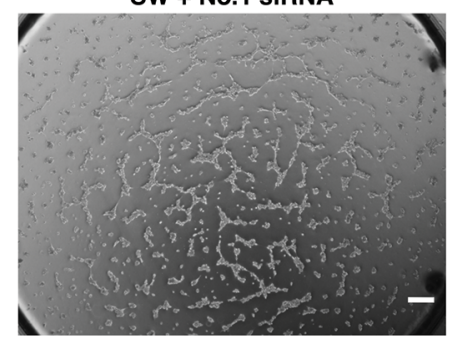

E
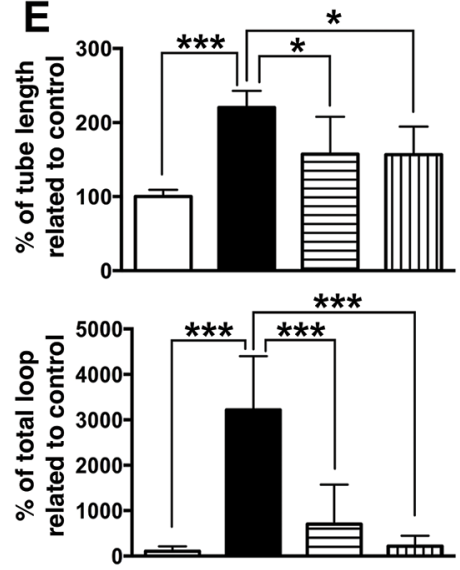

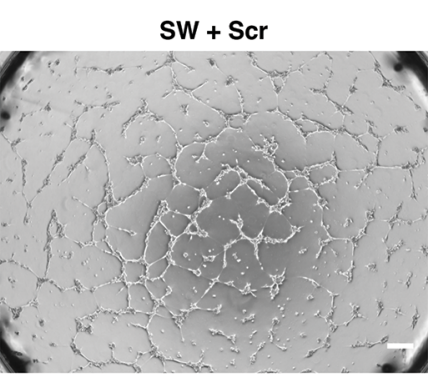

SW + No.2 siRNA
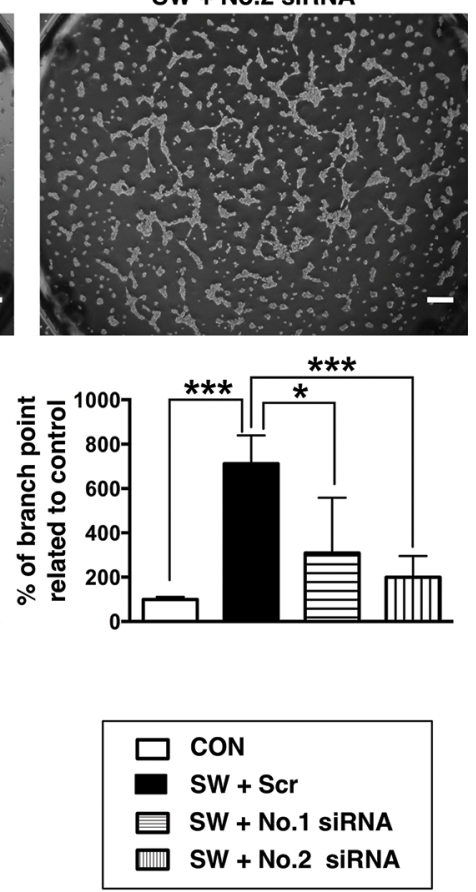

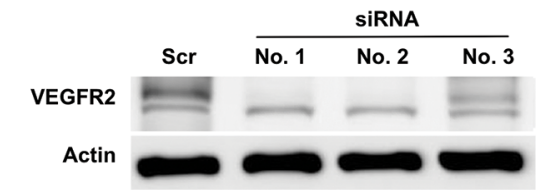

Figure 6. Importance of vascular endothelial growth factor receptor 2 (VEGFR2) in shock wave (SW)-induced angiogenesis. (A) Treatment with low-dose (5 $\mu \mathrm{mol} / \mathrm{L})$ (SW + Low SU5416) and high-dose (15 $\mu \mathrm{mol} / \mathrm{L})$ (SW + High SU5416) of SU5416 (VEGFR2 kinase inhibitor) in SW-treated HUVECs during tube formation assay. Morphology of tube-like structure at $6 \mathrm{~h}$ post seeding. (B) Parameters of tube formation assay, including tube length, branch point and the number of loops ( $n=7$ in each group). (C) Three different small interfering RNAs (siRNAs) for VEGFR2 silencing in HUVECs, evaluated by Western blot. (D) No. 1 and No. 2 siRNAs-transfected HUVECs applied in tube formation assay, and (E) Quantification ( $\mathrm{n}=6$ in each group). Data shown as mean \pm S.D. ${ }^{* * *} P<0.0005,{ }^{*} P<0.05$ determined by Student $t$ test.

$(8,10)$. However, we find that activation of VEGFR2-Akt-eNOS signaling pathway per se is insufficient to explain the increase in VEGFR2 protein expression following SW treatment without mRNA changes. Furthermore, both exogenous and endogenous nitric oxide (NO) have been reported to inhibit externalization of tissue transglutaminase (tTG) through attenuation of recycling endosomes in human aortic endothelial cells (33). We assume that recycling of endocytic VEGFR2 may be repressed by NO production so that VEGFR2 expression in the membrane fraction did not increase during the first few hours post-SW treatment. On the other hand, the mechanism of NO-mediated VEGFR2 recycling is still poorly understood.

Our findings demonstrated that VEGFR2 may undergo enhanced 
A

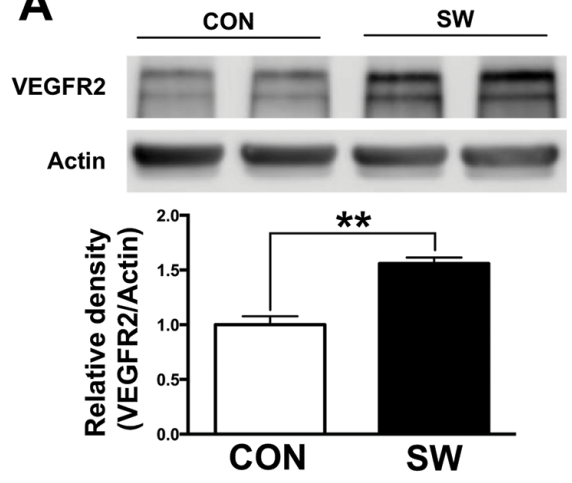

B

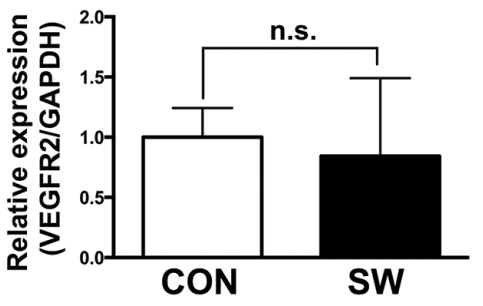

C

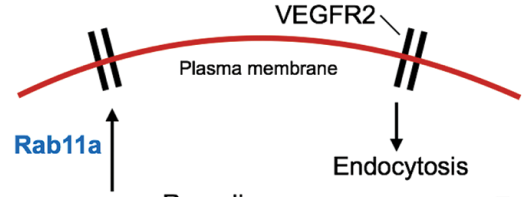

D

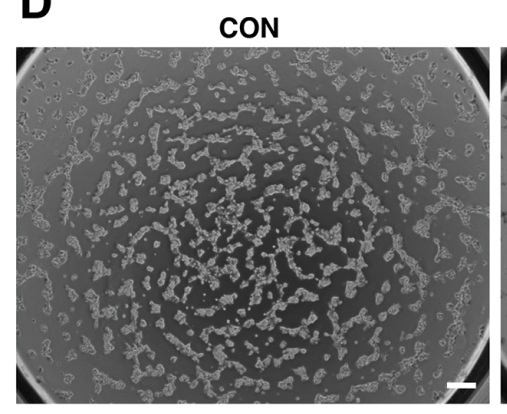

SW + No.2 SiRNA

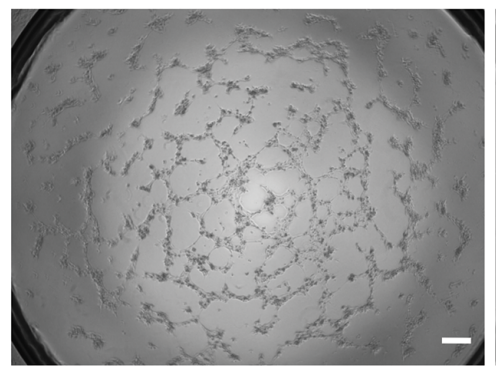

E
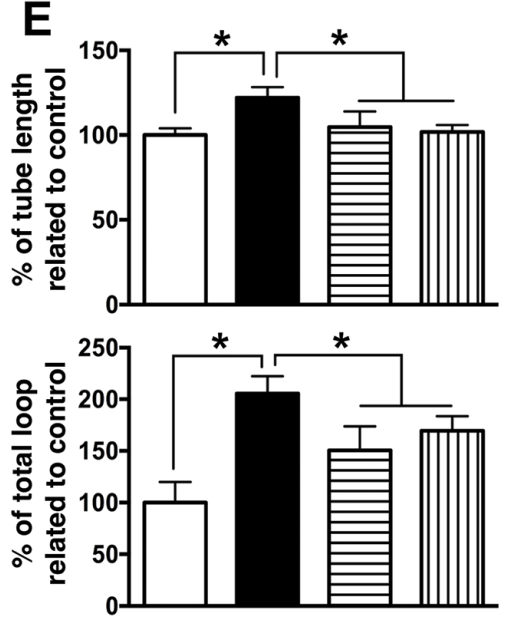

$S W+S c r$

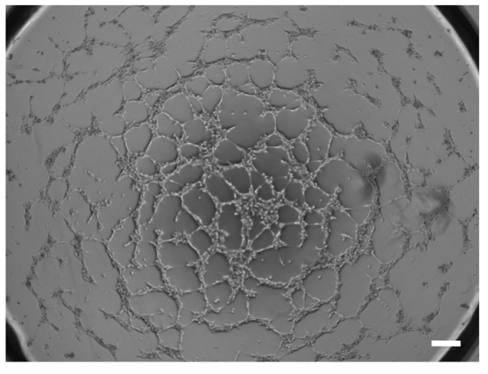

SW + No.3 SiRNA
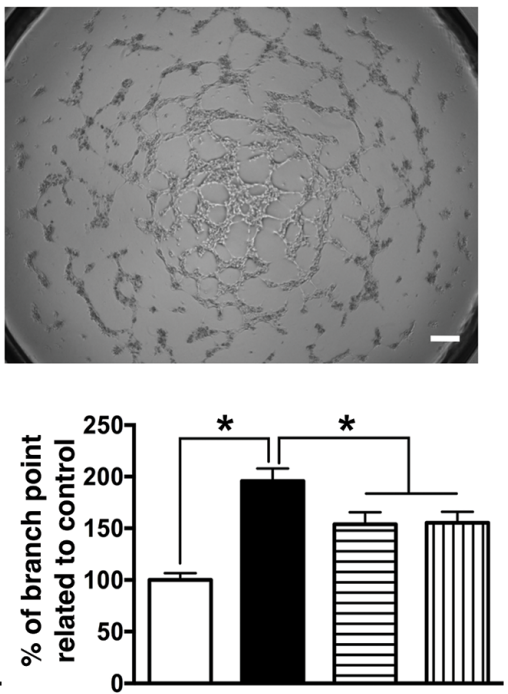

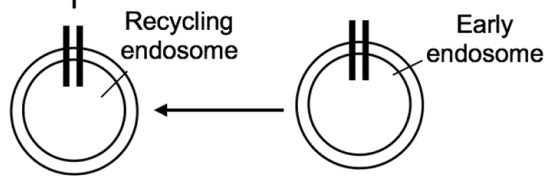

Rab11a

Scr siRNA

Actin
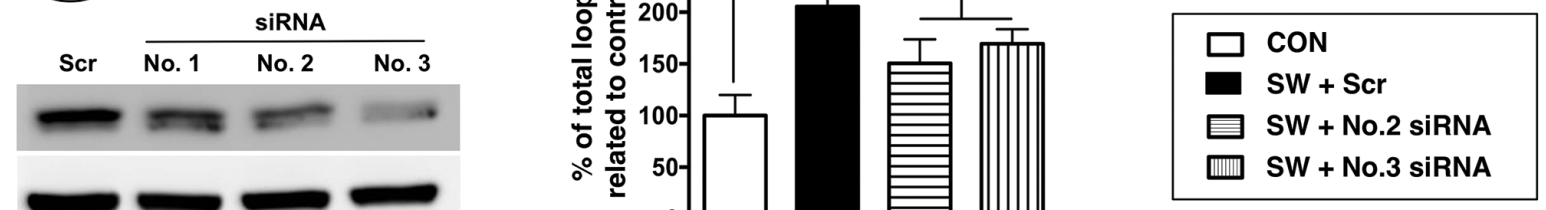

Figure 7. Expression of vascular endothelial growth factor receptor 2 (VEGFR2) in shock wave (SW)-induced angiogenesis. (A) Protein expression of VEGFR2 in HUVECs with (SW) and without (CON) SW treatment determined by Western blot ( $\mathrm{n}=4$ in each group). (B) Expression of VEGFR2 mRNA in HUVECs at the end of $28 \mathrm{~h}$ without (CON) and with SW treatment determined by RT-qPCR ( $n=3$ in each group). (C) Illustration showed the endocytic route of endothelial VEGFR2 from early endosome to recycling endosome and ship back to the plasma membrane through Rabl la. Rabl la silencing in HUVECs by three different small interfering RNAs (siRNAs) evaluated by Western blot. (D) Tube formation assay posttransfection of scramble (Scr), No.2, and No.3 siRNAs with SW compared with those in the control (CON) without SW treatment. (E) Changes in parameters of tube formation assay, including tube length, branch point and the number of loops ( $n=5$ in each group) post Rabl la silencing with No.2 and No.3 siRNAs. Data shown as mean \pm S.D. ${ }^{*} P<0.05$ determined by Student $t$ test.

endocytic recycling post-SW treatment. Recycling of membrane receptors is generally believed to be attained through two pathways. While one is the fast pathway that involves the endocytosis of membrane receptors with the formation of early endosome that quickly fuses with cell membrane (within minutes) with the presentation of receptors, again on the cell surface via Rab4a, the other slow recycling pathway (within hours) comprises transformation of early endosome into recycling endosome before receptor presentation on cell membrane. In this picture, Rab11a has been reported to be associated with slow recycling trafficking $(19,34)$, the silencing of which has been demonstrated to suppress 

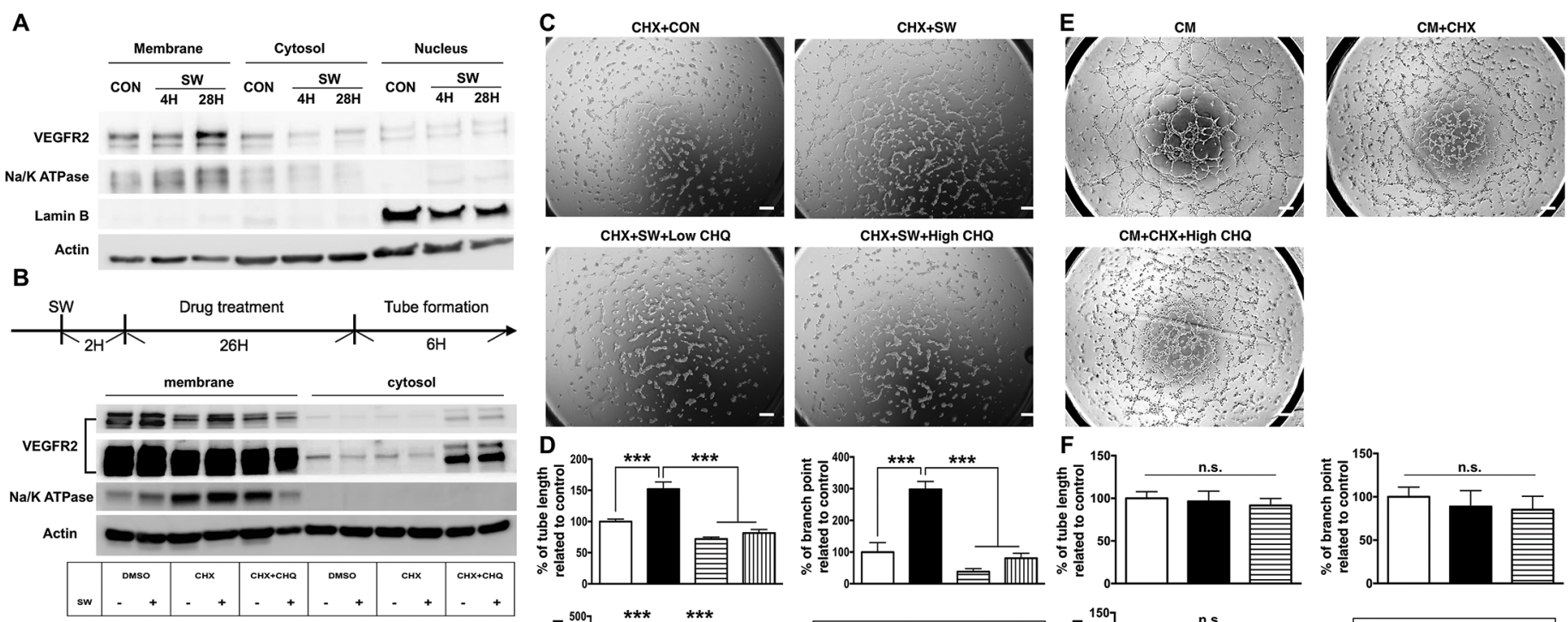

$\mathrm{CM}+\mathrm{CHX}+\mathrm{High} \mathrm{CHQ}$
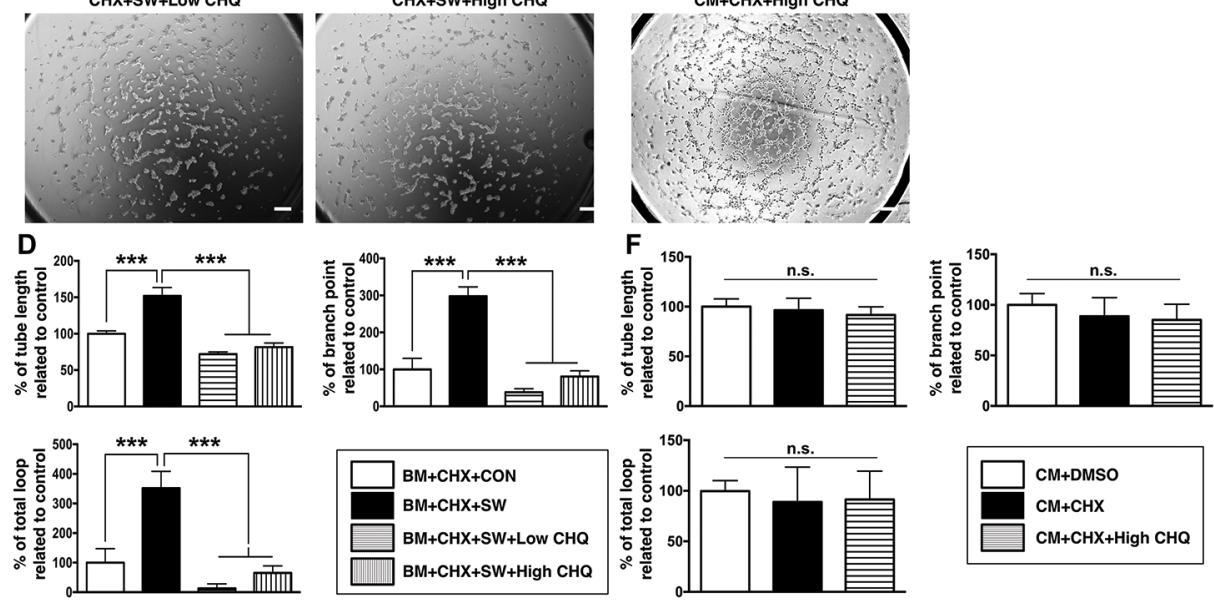

Figure 8. Endocytic recycling of vascular endothelial growth factor receptor 2 (VEGFR2) post-shock wave (SW) treatment. (A) VEGFR2, Na/K ATPase (marker of membrane protein), Lamin B (marker of nucleus protein) and actin in protein fraction of HUVECs (including membrane, cytosol and nucleus parts) at the end of the first 4 and $28 \mathrm{~h}$ post-SW treatment compared with control (CON) assessed by Western blot. (B) The workflow noted three time points for SW, drug treatment and tube formation. Protein expressions of VEGFR2 (with short and long exposure time), Na/K ATPase and actin in HUVECs (fractionized into membrane and cytosol parts) assessed by Western blot $26 \mathrm{~h}$ following DMSO, cycloheximide (CHX) and chloroquine (CHQ) treatment with and without SW administration. (C) Tube formation assay for HUVECs $26 \mathrm{~h}$ post being treated with cycloheximide (CHX) and chloroquine (CHQ, $5 \& 20 \mu \mathrm{mol} / \mathrm{L}$ ) in basal medium (BM, without FBS and growth factors) with and without SW treatment. (D) Parameters of tube formation assay, including tube length, branch point and the number of loops ( $n=6$ in each group). (E) Tube formation assay for HUVECs $26 \mathrm{~h}$ after being treated with DMSO, cycloheximide ( $\mathrm{CHX}$ ) and high concentration of chloroquine (CHQ, 20 $\mu \mathrm{mol} / \mathrm{L}$ ) in complete medium (CM, that is, contained FBS and growth factors). (F) Parameters of tube formation assay, including tube length, branch point and the number of loops ( $n=5$ in each group). Data shown as mean \pm S.D. ${ }^{* * *} P<0.0001$ determined by Student $t$ test.

VEGFA-stimulated migration and proliferation, as well as tube formation in HUVECs (35). Consistently, we found that silencing of Rab11a also reduced tube formation post-SW treatment without exogenous VEGFA activation, implying that Rab11a-mediated slow recycling pathway is associated with SW treatment. In our vessel sprouting (ex vivo) and limb ischemia (in vivo) models, we also observed that SW-induced angiogenesis was prolonged more than a week. Taken together, we assumed that SW-induced VEGFR2 recycling may be achieved mainly by slow endosomal recycling through Rab11a instead of rapid endosomal recycling via Rab4a $(36,37)$.
In the current study, we also displayed that $\mathrm{Na} / \mathrm{K}$ ATPase, which acted as a membrane-specific marker, was also increased post-SW treatment. Interestingly, an effect of laminar fluid shear stress has been reported to be similar to low-energy SW treatment (14), and treatment of laminar fluid shear stress induced a great surface expression of NA/K ATPase on MPT cells by the stimulation of exocytic pathway (38). Hence, we speculated that expression of $\mathrm{Na} / \mathrm{K}$ ATPase might also be regulated by SW treatment through endosome recycling, suggesting further investigation is required.

The present study has several limitations. First, this study explored only the angiogenic effect of SW treatment on endothelial cells in vitro. Therefore, the SW effect on adjacent stromal cells is still unclear, so the establishment of an endothelial-stromal cells co-culture model for this issue is necessary. Second, there has not been tangible evidence showing the linkage between nitric oxide production and VEGFR2 recycling post-SW treatment. Third, although the results are promising, the mechanism underlying SW-induced endosome recycling remains to be elucidated.

\section{CONCLUSION}

The current study demonstrated that VEGFR2 activation and recycling were 
augmented by SW treatment in endothelial cells, and were associated with enhanced and prolonged angiogenesis. Our findings not only delineated a novel mechanism underlying SW treatment, but also unveil the possibility of novel drug development for the treatment of ischemic diseases through enhancement of VEGFR2 recycling.

\section{ACKNOWLEDGMENTS}

This study was supported by a program grant from Chang Gung Memorial Hospital (Grant number: CMRPG8D0431).

\section{DISCLOSURE}

The authors declare they have no competing interests as defined by Molecular Medicine, or other interests that might be perceived to influence the results and discussion reported in this paper.

\section{REFERENCES}

1. Langer PR. (2015) Two emerging technologies for Achilles tendinopathy and plantar fasciopathy. Clin. Podiatr. Med. Surg. 32:183-93.

2. Wang CJ. (2012) Extracorporeal shockwave therapy in musculoskeletal disorders. J. Orthop. Surg. Res. 7:11.

3. Mani-Babu S, Morrissey D, Waugh C, Screen H, Barton C. (2015) The effectiveness of extracorporeal shock wave therapy in lower limb tendinopathy: a systematic review. Am. J. Sports Med. 43:752-61.

4. Wang CJ, Wang FS, Yang KD, Weng LH, Ko JY. (2006) Long-term results of extracorporeal shockwave treatment for plantar fasciitis. Am. J. Sports. Med. 34:592-6.

5. Gruenwald I, Appel B, Kitrey ND, Vardi Y. (2013) Shockwave treatment of erectile dysfunction. Ther. Adv. Urol. 5:95-9.

6. Gruenwald I, Appel B, Vardi Y. (2012) Low-intensity extracorporeal shock wave therapy-a novel effective treatment for erectile dysfunction in severe ED patients who respond poorly to PDE5 inhibitor therapy. J. Sex. Med. 9:259-64.

7. Goertz O, von der Lohe L, Lauer H, et al. (2014) Repetitive extracorporeal shock wave applications are superior in inducing angiogenesis after full thickness burn compared to single application. Burns. 1365-74.

8. Sheu JJ, Lee FY, Yuen CM, et al. (2015) Combined therapy with shock wave and autologous bone marrow-derived mesenchymal stem cells alleviates left ventricular dysfunction and remodeling through inhibiting inflammatory stimuli, oxidative stress \& enhancing angiogenesis in a swine myocardial infarction model. Int. J. Cardiol. 193:69-83.
9. Nishida T, Shimokawa H, Oi K, et al. (2004) Extracorporeal cardiac shock wave therapy markedly ameliorates ischemia-induced myocardial dysfunction in pigs in vivo. Circulation. 110:3055-61.

10. Yeh KH, Sheu JJ, Lin YC, et al. (2012) Benefit of combined extracorporeal shock wave and bone marrow-derived endothelial progenitor cells in protection against critical limb ischemia in rats. Crit. Care Med. 40:169-77.

11. Aicher A, Heeschen C, Sasaki K, Urbich C, Zeiher AM, Dimmeler S. (2006) Low-energy shock wave for enhancing recruitment of endothelial progenitor cells: a new modality to increase efficacy of cell therapy in chronic hind limb ischemia. Circulation. 114:2823-30.

12. Jin $Z G$, Ueba $H$, Tanimoto $T$, Lungu AO, Frame MD, Berk BC. (2003) Ligand-independent activation of vascular endothelial growth factor receptor 2 by fluid shear stress regulates activation of endothelial nitric oxide synthase. Circ. Res. 93:354-63.

13. Fukumura D, Gohongi T, Kadambi A, et al. (2001) Predominant role of endothelial nitric oxide synthase in vascular endothelial growth factor-induced angiogenesis and vascular permeability. Proc. Natl. Acad. Sci. U. S. A. 98:2604-9.

14. Ha CH, Kim S, Chung J, An SH, Kwon K. (2013) Extracorporeal shock wave stimulates expression of the angiogenic genes via mechanosensory complex in endothelial cells: mimetic effect of fluid shear stress in endothelial cells. Int. J. Cardiol. 168:4168-77.

15. Jopling HM, Howell GJ, Gamper N, Ponnambalam S. (2011) The VEGFR2 receptor tyrosine kinase undergoes constitutive endosome-to-plasma membrane recycling. Biochem. Biophys. Res. Commun. 410:170-6.

16. Ballmer-Hofer K, Andersson AE, Ratcliffe LE, Berger P. (2011) Neuropilin-1 promotes VEGFR-2 trafficking through Rab11 vesicles thereby specifying signal output. Blood. 118:816-26.

17. Cavalli V, Vilbois F, Corti M, et al. (2001) The stress-induced MAP kinase p38 regulates endocytic trafficking via the GDI:Rab5 complex. Mol. Cell. 7:421-32.

18. Raghavan V, Rbaibi Y, Pastor-Soler NM, Carattino MD, Weisz OA. (2014) Shear stress-dependent regulation of apical endocytosis in renal proximal tubule cells mediated by primary cilia. Proc. Natl. Acad. Sci. U. S. A. 111:8506-11.

19. Ullrich O, Reinsch S, Urbé S, Zerial M, Parton RG. (1996) Rab11 regulates recycling through the pericentriolar recycling endosome. J. Cell Biol. 135:913-24

20. Khalil IA, Kogure K, Akita H, Harashima H. (2006) Uptake pathways and subsequent intracellular trafficking in nonviral gene delivery. Pharmacol. Rev. 58:32-45.

21. Yuyama K, Yamamoto N, Yanagisawa K (2006) Chloroquine-induced endocytic pathway abnormalities: Cellular model of GM1 ganglioside-induced Abeta fibrillogenesis in Alzheimer's disease. FEBS Lett. 580:6972-6.
22. Cai S, Khoo J, Mussa S, Alp NJ, Channon KM. (2005) Endothelial nitric oxide synthase dysfunction in diabetic mice: importance of tetrahydrobiopterin in eNOS dimerisation. Diabetologia. 48:1933-40.

23. Gershater MA, Löndahl M, Nyberg $\mathrm{P}$, et al. (2009) Complexity of factors related to outcome of neuropathic and neuroischaemic/ischaemic diabetic foot ulcers: a cohort study. Diabetologia. 52:398-407.

24. Moretti B, Notarnicola A, Maggio G, et al. (2009) The management of neuropathic ulcers of the foot in diabetes by shock wave therapy. BMC Musculoskelet. Disord. 10:54.

25. Omar MT, Alghadir A, Al-Wahhabi KK, AlAskar AB. (2014) Efficacy of shock wave therapy on chronic diabetic foot ulcer: a single-blinded randomized controlled clinical trial. Diabetes Res. Clin. Pract. 106:548-54.

26. Olsson AK, Dimberg A, Kreuger J, Claesson-Welsh L. (2006) VEGF receptor signalling - in control of vascular function. Nat. Rev. Mol. Cell. Biol. 7:359-71.

27. You J, Reilly GC, Zhen X, et al. (2001) Osteopontin gene regulation by oscillatory fluid flow via intracellular calcium mobilization and activation of mitogen-activated protein kinase in MC3T3-E1 osteoblasts. J. Biol. Chem. 276:13365-71.

28. Fleming I, Busse R. (1999) Signal transduction of eNOS activation. Cardiovasc. Res. 43:532-41.

29. Uwatoku T, Ito K, Abe K, et al. (2007) Extracorporeal cardiac shock wave therapy improves left ventricular remodeling after acute myocardial infarction in pigs. Coron. Artery Dis. 18:397-404.

30. Oi K, Fukumoto Y, Ito K, et al. (2008) Extracorporeal shock wave therapy ameliorates hindlimb ischemia in rabbits. Tohoku J. Exp. Med. 214:151-8.

31. Fukumoto $\mathrm{Y}$, Ito A, Uwatoku T, et al. (2006) Extracorporeal cardiac shock wave therapy ameliorates myocardial ischemia in patients with severe coronary artery disease. Coron. Artery Dis. 17:63-70.

32. Vasyuk YA, Hadzegova AB, Shkolnik EL, et al. (2010) Initial clinical experience with extracorporeal shock wave therapy in treatment of ischemic heart failure. Congest. Heart Fail. 16:226-30.

33. Santhanam L, Berkowitz DE, Belkin AM. (2011) Nitric oxide regulates non-classical secretion of tissue transglutaminase. Commun. Integr. Biol. 4:584-6.

34. Lock JG, Stow JL. (2005) Rab11 in recycling endosomes regulates the sorting and basolateral transport of E-cadherin. Mol. Biol. Cell. 16:1744-55.

35. Jopling HM, Odell AF, Pellet-Many C, et al. (2014) Endosome-to-plasma membrane recycling of VEGFR2 receptor tyrosine kinase regulates endothelial function and blood vessel formation. Cells. 3:363-85.

36. Perrin L, Laura P, Lacas-Gervais S, et al. (2013) Rab4b controls an early endosome sorting event by interacting with the $\gamma$-subunit of the clathrin adaptor complex 1. J. Cell. Sci. 126:4950-62. 
VEGFR2 ACTIVATION/RECYCLING BY SHOCK WAVE

37. Sönnichsen B, De Renzis S, Nielsen E, Rietdorf J, Zerial M. (2000) Distinct membrane domains on endosomes in the recycling pathway visualized by multicolor imaging of Rab4, Rab5, and Rab11. J. Cell Biol. 149:901-14

38. Duan $Y$, Weinstein AM, Weinbaum S, Wang T. (2010) Shear stress-induced changes of membrane transporter localization and expression in mouse proximal tubule cells. Proc. Natl. Acad. Sci. U. S. A. 107:21860-5.

Cite this article as: Huang TH, et al. (2016) Shock wave therapy enhances angiogenesis through VEGFR2 activation and recycling. Mol. Med. 22:850-62. 